Publ. RIMS, Kyoto Univ.

18 (1982), 241-263

\title{
Actions of Semigroups on Borel Spaces and von Neumann Algebras of Groupoids of Type $\mathrm{II}_{1}$
}

By

\author{
Moto O’UCHI*
}

\section{$\S 0 . \quad$ Introduction}

The natural action of $S L(n, \mathbf{Z})$ on the $n$-dimensional torus $\mathbb{T}^{n}$ with the Lebesgue measure is non-amenable (c.f. [13]) and it is important to study the groupoid $\mathbf{T}^{n} \times S L(n, \mathbf{Z})$ and its associated von Neumann algebra. For $k \in \mathbb{Z}$ and the unit matrix $I$ of $S L(n, \mathbb{Z}), k I$ defines naturally a Borel map of $\mathbf{T}^{n}$ onto itself, which is, in an appropriate sense, compatible with the action of $S L(n, \mathbb{Z})$. We pick up this property and study its effect on the groupoid and on the associated von Neumann algebra. In Section 1, we introduce the notion of an action of a semigroup on a Borel space by generalizing the above property (Definition 1.3). From an action of a semigroup $B$ on a Borel space, we construct a von Neumann algebra $\operatorname{End}_{A}(H)$ according to [2] and a homomorphism $\Phi$ of the semigroup $B$ into the semigroup of injective endomorphisms of $\operatorname{End}_{A}(H)$. Then we study, in Theorem 1.10, a necessary condition for two algebras $\Phi_{b_{1}}\left(\operatorname{End}_{\Lambda}(H)\right)$ and $\Phi_{b_{2}}\left(\operatorname{End}_{A}(H)\right) \quad\left(b_{1}, b_{2} \in B\right)$ to be inner conjugate in $\operatorname{End}_{A}(H)$ (two subalgebras $\mathfrak{M}_{1}$ and $\mathfrak{M}_{2}$ of a von Neumann algebra $\mathfrak{M}$ are said to be inner conjugate in $\mathfrak{M}$ if there exists an inner automorphism $\alpha$ of $\mathfrak{M}$ such that $\left.\alpha\left(\mathfrak{M}_{1}\right)=\mathfrak{M}_{2}\right)$. In Section 2, we study a sufficient condition for $\Phi_{b_{1}}\left(\operatorname{End}_{\Lambda}(H)\right)$ and $\Phi_{b_{2}}\left(\operatorname{End}_{\Lambda}(H)\right)\left(b_{1}, b_{2} \in B\right)$ not to be conjugate by any automorphism of $\operatorname{End}_{\Lambda}(H)$ (Corollary 2.9). In Section 3, we apply the general argument in Sections 2 and 3 to the action of $S L(n, \mathbb{Z})$ on $\mathbb{T}^{n}$. Let $\mathfrak{M}_{n}$ be the factor of type $\mathrm{II}_{1}$ obtained from the action of $S L(n, \mathbb{Z})$

Communicated by H. Araki, March 27, 1981.

* Department of Mathematics, Faculty of Science, Ehime University, Matsuyama, 790, Japan. 
by the group-measure space construction. Then we construct a family $\left\{\mathfrak{N}_{k}^{n}\right\}_{k \in \mathbf{N}}$ of subalgebras of $\mathfrak{M}_{n}$ such that $\mathfrak{N}_{k}^{n}$ is spatially isomorphic to $\mathfrak{M}_{n} \otimes \mathbf{C}_{k^{n}}$, where $\mathbf{C}_{k^{n}}$ is the algebra of scalar operators on the $k^{n}$-dimensional Hilbert space. It is also shown that, for $k \geq 2, \cap_{i=1}^{\infty} \mathfrak{N}_{k i}^{n}$ is isomorphic to $\lambda(S L(n, \mathbf{Z})) "$, where $\lambda$ is the right regular representation of $S L(n, \mathbf{Z})$ (Theorem 3.2). If $\lambda(S L(n, \mathbb{Z}))$ " is considered as a subalgebra of $\mathfrak{M}_{n}$ by the natural embedding, then $\lambda(S L(n, \mathbf{Z}))^{\prime \prime}$ can be characterized as a fixed point algebra of a certain endomorphism of $\mathfrak{M}_{n}$.

The author would like to express his hearty thanks to Professor O. Takenouchi for constant encouragement and helpful suggestions.

\section{$\S$ 1. Action of a Semigroup on a Borel Space}

In this section, we introduce the notion of an action of a semigroup on a Borel space and study the associated groupoid and its von Neumann algebra. First of all, we establish definitions and notations which will be used in this paper. As for groupoids, we use definitions and notations in [2] (see also [10]). For a measurable groupoid $G$, the left (resp. right) unit $r \gamma^{-1}$ (resp. $\gamma^{-1} \gamma$ ) of $\gamma \in G$ is denoted by $r(\gamma)$ (resp. $s(\gamma)$ ). The unit space $G^{(0)}$ of $G$ is the Borel space defined by $G^{(0)}=r(G)$, and the set of all composable elements is the Borel space $G^{(2)}=\left\{\left(\gamma_{1}, \gamma_{2}\right) \in G\right.$ $\left.\times G ; s\left(\gamma_{1}\right)=r\left(\gamma_{2}\right)\right\}$. We write $G^{y}$ for $r^{-1}(y)\left(y \in G^{(0)}\right)$. For $x, y \in G^{(0)}$, $x$ and $y$ are equivalent with respect to $G$ (or $G$-equivalent) if and only if there exists an element $\gamma \in G$ such that $s(\gamma)=x$ and $r(\gamma)=y$. If $x$ and $y$ are equivalent, we write $x \sim y$. For a Borel subset $E$ of $G^{(0)}$, the saturation $[E]$ of $E$ with respect to $G$ (or the $G$-saturation of $E$ ) is the set $\left\{y \in G^{(0)}\right.$; for some $\left.x \in E, y \sim x\right\}$. The reduction of $G$ to $E$ is the measurable groupoid $G \mid E$ defined by $G \mid E=\{r \in G ; r(\gamma) \in E$ and $s(\gamma)$ $\in E\}$. Let $\mathcal{E}^{+}(G)$ be the set of proper transverse functions on $G[2$, p. 37, Definition 2], and $\Lambda$ be a transverse measure of module $\delta$ on $G$ $\left[2\right.$, p. 41 , Definition 1]. For a saturated Borel set $E$ of $G^{(0)}, E$ is $\Lambda$ null if and only if there exists a faithful $\nu \in \mathcal{E}^{+}(G)$ such that $A_{\nu}(E)$ $=0$ [2, p. 48, Proposition 8]. If $G$ is discrete [2, p. 40], there exists $\nu \in \mathcal{E}^{+}(G)$ such that $\nu^{y}(\{\gamma\})=1$ for all $\gamma \in G^{y}$ and all $y \in G^{(0)}$. We call this $\nu$ the transverse function of counting measures. 
Let $A$ be a group, $B$ be a sub-semigroup of $A$ and $C$ be a countable discrete normal subgroup of $A$ which is contained in $B$. For a standard Borel space $S, \mathscr{B}(S)$ denotes the set of Borel maps of $S$ onto $S$ which send Borel subsets of $S$ to Borel subsets of $S$. For $\phi, \psi \in$ $\mathscr{B}(S)$, the product $\phi \circ \psi$ of $\phi$ and $\psi$ is defined by $\phi \circ \psi(x)=\psi(\phi(x))(x \in S)$. Then $\mathscr{B}(S)$ becomes a semigroup. Let $\alpha$ be a homomorphism of the semigroup $B$ into the semigroup $\mathscr{B}(S)$ such that $\alpha(e)$ is the identity map on $S$, where $e$ is the unit of $A$. We write $x b$ instead of $\alpha(b)(x) \quad(x$ $\in S, b \in B)$, Then $S$ is a Borel $C$-space with respect to the restriction of the above action to $C$ and $G=S \times C$ becomes a measurable groupoid, that is, elements $\left(x, c_{1}\right)$ and $\left(y, c_{2}\right)$ of $G$ are composable if and only if $y=x c_{1}$ and we have $\left(x, c_{1}\right)\left(x c_{1}, c_{2}\right)=\left(x, c_{1} c_{2}\right)$. Note that $G^{(0)}=\{(x, e)$; $x \in S\}$ is identified with $S$. We assume that the action of $C$ on $S$ is free, that is, for every $x \in S,\{c \in C ; x c=x\}=\{e\}$. For $x \in S$, the saturation $[x]$ of $x$ with respect to $G$ is the orbit of $x$ under the action of $C$, i.e. $[x]=\{x c ; c \in C\}$.

Lemma 1. 1. If $b$ is an element of $B$, then $[x b]=[x] b$ for every $x \in S$, where $[x] b=\{y b ; y \in[x]\}$.

Proof. Since $C$ is a normal subgroup of $A$, for $b \in B$ and $c \in C$, there exist $c_{1}, c_{2} \in C$ such that $b c=c_{1} b$ and $c b=b c_{2}$. Then we have $x b c$ $=x c_{1} b$ and $x c b=x b c_{2}$ for $x \in S$. As $c$ is arbitrary, this implies that $[x b] \subset[x] b$ and $[x] b \subset[x b]$.

Q.E.D.

We define an equivalence relation $\widetilde{b}$ on $S$ by the following; for $x$, $y \in S, x_{\widetilde{b}} y$ if and only if $x b \sim y b$, where $\sim$ denotes the equivalence relation with respect to $G$. Put $G \cdot b=\left\{(x, y) \in S \times S ; x_{\widetilde{b}} y\right\}$, Then $G \cdot b$ becomes a measurable groupoid, that is, elements $\left(x_{1}, y_{1}\right)$ and $\left(x_{2}, y_{2}\right)$ of $G \cdot b$ are composable if and only if $y_{1}=x_{2}$ and we have $\left(x_{1}, y_{1}\right)\left(y_{1}, y_{2}\right)$ $=\left(x_{1}, y_{2}\right)$. Note that $G$ can be considered as a subgroupoid of $G \cdot b$ by the injection $(x, c) \mapsto(x, x c) \quad((x, c) \in G)$. The saturation of $E$ with respect to $G \cdot b$ is denoted by $[E]_{b}$.

Lemma 1.2. (i) If $E$ is a saturated set with respect to $G$, 
then, for every $b, E b$ and $E b^{-1}$ are saturated with respect to $G$, where $E b^{-1}=\{x \in S ; x b \in E\}$.

(ii) For $b \in B$ and $x \in S$, put $F=\{y \in S ; y b=x b\}$. Then $[x]_{b}$ is a disjoint union of $\{[y]\}_{y \in F}$.

Proof. (i) By Lemma 1.1 , it is clear that $[E b]=E b$. For $x$ $\in\left[E b^{-1}\right]$, there exists $y \in E b^{-1}$ such that $x \sim y$. As we have $x b \sim y b$ and $y b \in E, x b$ belongs to $E$. It follows that $\left[E b^{-1}\right]=E b^{-1}$.

(ii) It is clear that $\cup_{y \in F}[y] \subset[x]_{b}$. For $y \in[x]_{b}$, there exists $c \in C$ such that $x b=y b c$. Then, for some $c_{1} \in C$, we have $x b=y c_{1} b$ and $y c_{1}$ belongs to $F$. Thus we have $[x]_{b} \subset \cup_{y \in F}[y]$. Now, suppose that, for $y_{1}, y_{2} \in F, y_{1}$ belongs to $\left[y_{2}\right]$. There exist $c, c_{1} \in C$ such that $y_{1}$ $=y_{2} c$ and $c b=b c_{1}$. We have

$$
x b=y_{1} b=y_{2} b c_{1}=x b c_{1} .
$$

Since the action of $C$ is free, this implies that $c=c_{1}=e$. It follows that $y_{1}=y_{2}$, and this means that $\{[y]\}_{y \in F}$ are disjoint.

Q.E.D.

Definition 1.3. Let $(A, B, G)$ be as above and $\Lambda$ be a $\sigma$-finite transverse measure on $G$. A quartet $(A, B, G, \Lambda)$ is called an action of $B$ on $S$ if it satisfies the following condition; for a $G$-saturated Borel set $E$ of $S$, if $E$ is $A$-null, then $E b$ and $E b^{-1}$ are $A$-null for every $b \in B$.

From now on we assume that $(A, B, G, A)$ is an action of $B$ on $S$. Let $\nu$ be the transverse function of counting measures and $(H, L)=(H$, $\left.L^{\nu}\right)$ be the left regular representation of $G[2$, p. 74], that is, the field $H=\left(H_{y}\right)_{y \in S}$ of Hilbert spaces is defined by $H_{y}=L^{2}\left(G^{y}, \nu^{y}\right)$ and, for $\gamma$ $\in G$, the isometry $L(\gamma)$ of $H_{s(r)}$ onto $H_{r(r)}$ is defined by

$$
(L(\gamma) f)\left(\gamma^{\prime}\right)=f\left(\gamma^{-1} \gamma^{\prime}\right) \quad \text { for } \quad f \in H_{s(r)} \quad \text { and } \quad \gamma^{\prime} \in G^{r(r)} .
$$

Note that, for every $y \in S, H_{y}$ is isomorphic to $l^{2}(C)$ since $\nu^{y}$ is the counting measure on $G^{y}$. By $[2$, p. 86 , Theorem 4$]$, there is the von Neumann algebra $\operatorname{End}_{\Lambda}(H)$ associated with $(H, L)$. Elements of $\operatorname{End}_{\Lambda}(H)$ are classes of elements of $\operatorname{End}_{G}(H)$ modulo the equality $\Lambda$-almost everywhere, where $\operatorname{End}_{G}(H)$ is the set of intertwining operators of $(H, L) \quad[2$, p. 84, Definition 1$]$. We 'sometimes consider elements of 
$\operatorname{End}_{A}(H)$ as elements of $\operatorname{End}_{G}(H)$. Let $b$ be an element of $B$. For every $x \in S$, define an isometry $\phi_{x b, x}=\phi_{x b, x}^{b}$ of $H_{x}$ onto $H_{x b}$ by

$$
\phi_{x b, x}(f)(x b, c)=f\left(x, b c b^{-1}\right)
$$

for every $f \in H_{x}$ and $(x b, c) \in G^{x b}$.

Lemma 1. 4. If $c$ is an element of $C$, then

$$
L\left(x b, b^{-1} c b\right) \circ \phi_{x c b, x c}=\phi_{x b, x} \circ L(x, c)
$$

for every $x \in S$.

Proof. Note that, as $C$ is a normal subgroup of $A, b c b^{-1}$ and $b^{-1} c b$ belong to $C$ for every $b \in B$. Since the action of $B$ is associative, we have

$$
(x b)\left(b^{-1} c b\right)=x\left(b b^{-1} c b\right)=x c b .
$$

Thus the right unit of $\left(x b, b^{-1} c b\right)$ is $x c b$. For every $f \in H_{x c}$ and $c^{\prime}$ $\in C$, we have

$$
\begin{aligned}
& L\left(x b, b^{-1} c b\right) \circ \phi_{x c b, x c}(f)\left(x b, c^{\prime}\right) \\
& \quad=f\left(x c, c^{-1} b c^{\prime} b^{-1}\right) \\
& =\phi_{x b, x} \circ L(x, c)(f)\left(x b, c^{\prime}\right) .
\end{aligned}
$$

Proposition 1.5. For an element $T=\left(T_{x}\right)_{x \in S}$ of $\operatorname{End}_{4}(H)$, put

$$
\Phi_{b}(T)_{x}=\phi_{x b, x}^{-1} \circ T_{x b} \circ \phi_{x b, x} \text { for every } x \in S,
$$

then $\Phi_{b}(T)=\left(\Phi_{b}(T)_{x}\right)_{x \in S}$ is an element of $\operatorname{End}_{\Lambda}(H)$.

Proof. Define a Borel structure for the field $H^{\prime}=\left(H_{x b}\right)_{x \in S}$ of Hilbert spaces by the following (c.f. [3, p. 142, Definition 1]); if $\xi^{i}$ $=\left(\xi_{x}^{i}\right) \quad(i=1,2, \cdots)$ is a fundamental sequence for the Borel structure of $H$, then $\left(\xi_{x b}^{i}\right)_{x \in S}(i=1,2, \cdots)$ is a fundamental sequence for $H^{\prime}$. Let $f$ be a Borel function on $G$ such that $\int|f|^{2} d \nu^{x}<+\infty$ for all $x \in S$. The restriction of $f$ to $G^{x}$ is denoted by $f_{x}$. Note that $\left(f_{x}\right)$ is a Borel section of $H$ and the Borel structure of $H$ is determined by the set of 
sections of this form. Then $\left(\phi_{x b, x}\left(f_{x}\right)\right)_{x \in S}$ is a Borel section of $H^{\prime}$. Since $\left(T_{x b}\right)_{x \in S}$ is a Borel field for $H^{\prime}$, for Borel sections $\left(f_{x}\right)$ and $\left(g_{x}\right)$ of $H$ of the above form, the function

$$
x \mapsto\left\langle\Phi_{b}(T)_{x} f_{x}, g_{x}\right\rangle=\left\langle T_{x b} \phi_{x b, x}\left(f_{x}\right), \phi_{x b, x}\left(g_{x}\right)\right\rangle
$$

is Borel. This implies that $\Phi_{b}(T)$ is a Borel field of operators for $H$.

Let $\gamma=\left(x, c_{0}\right)$ be an element of $G$. For $(x, c) \in G$ and $f \in H_{x c_{0}}$, we have

$$
\begin{aligned}
(L(\gamma) & \left.\Phi_{b}(T)_{x c_{0}} f\right)(x, c) \\
= & \left(T_{x c_{0} b} \circ \phi_{x c_{0} b, x c_{0}}(f)\right)\left(x c_{0} b, b^{-1} c_{0}^{-1} c b\right) \\
= & \left(L\left(x b, b^{-1} c_{0} b\right) \circ T_{x c_{0} b} \circ \phi_{x c_{0} b, x c_{0}}(f)\right)\left(x b, b^{-1} c b\right) \\
= & \left(T_{x b} \circ L\left(x b, b^{-1} c_{0} b\right) \circ \phi_{x c_{0} b, x c_{0}}(f)\right)\left(x b, b^{-1} c b\right) .
\end{aligned}
$$

By Lemma 1.4, we have

$$
\begin{aligned}
& \left(L(\gamma) \Phi_{b}(T)_{x c_{0}} f\right)(x, c) \\
& \quad=T_{x b} \circ \phi_{x b, x} \circ L\left(x, c_{0}\right)(f)\left(x b, b^{-1} c b\right) \\
& \quad=\left(\Phi_{b}(T)_{x} \circ L(\gamma) f\right)(x, c) .
\end{aligned}
$$

It follows that $\Phi_{b}(T)$ is an intertwining operator of $(H, L)$ to itself. If $T$ and $T^{\prime}$ are intertwining operators of $(H, L)$ which coincide with each other $\Lambda$-almost everywhere, then $\Phi_{b}(T)$ and $\Phi_{b}\left(T^{\prime}\right)$ coincide with each other $\Lambda$-almost everywhere by the assumption of $(A, B, G, \Lambda)$, Therefore $\Phi_{b}(T)$ is well-defined as an element of $\operatorname{End}_{\Lambda}(H)$.

Q.E.D.

In the above proposition, we have constructed a map $\Phi_{b}$ of $\operatorname{End}_{A}(H)$ into $\operatorname{End}_{A}(H)$. The following lemma shows that $\Phi_{b}$ is an injective endomorphism of $\operatorname{End}_{A}(H)$ and that the map $b \mapsto \Phi_{b}$ is a homomorphism of the semigroup $B$ into the semigroup of endomorphisms of $\operatorname{End}_{A}(H)$.

Lemma 1.6. (i) If $b_{1}$ and $b_{2}$ are elements of $B$, then, for every $T \in \operatorname{End}_{\Lambda}(H)$,

$$
\Phi_{b_{1}} \circ \Phi_{b_{2}}(T)=\Phi_{b_{1} b_{2}}(T)
$$

(ii) If $c$ is an element of $C, \Phi_{c}$ is an inner automorphism of $\operatorname{End}_{\Lambda}(H)$. 
(iii) For $b \in B$, the map $\Phi_{b}$ is an isometric *-homomorphism of $\operatorname{End}_{\Lambda}(H)$ into $\operatorname{End}_{\Lambda}(H)$.

Proof. (i) It is clear by an easy calculation.

(ii) Define a unitary operator $U_{x}$ on $H_{x}$ by

$$
\left(U_{x} f\right)\left(x, c^{\prime}\right)=f\left(x, c^{\prime} c^{-1}\right) \quad \text { for } \quad f \in H_{x} \quad \text { and } \quad\left(x, c^{\prime}\right) \in G^{x} .
$$

Then $U=\left(U_{x}\right)$ is a unitary element of $\operatorname{End}_{A}(H)$, and we have $\phi_{x c, x}^{c}$ $=L(x, c)^{-1} U_{x}$ for every $x \in S$. It follows that $\Phi_{c}(T)=U^{-1} T U$ for every $T \in \operatorname{End}_{\Lambda}(H)$.

(iii) It is clear that $\Phi_{b}$ is a ${ }^{*}$-homomorphism. Let $T$ be an element of $\operatorname{End}_{\Lambda}(H)$. For $\alpha \in \mathbf{R}_{+}$, we set $E_{\alpha}(T)=\left\{x \in S\right.$; $\left.\left\|T_{x}\right\|>\alpha\right\}$, which is a saturated Borel set. We have $E_{\alpha}\left(\Phi_{b}(T)\right)=E_{\alpha}(T) b^{-1}$ by the equation $\left\|\Phi_{b}(T)_{x}\right\|=\left\|T_{x b}\right\|$. Thus $E_{\alpha}(T)$ is $\Lambda$-null if and only if $E_{\alpha}\left(\Phi_{b}(T)\right)$ is A-null. Recall that the norm of $T$ is defined by

$$
\|T\|_{\infty}=\inf \left\{\alpha \in \mathbb{R}_{+} ; E_{\alpha}(T) \text { is } \Lambda \text {-null }\right\}
$$

[2, p. 84, Definition 1]. Hence we have $\left\|\Phi_{b}(T)\right\|_{\infty}=\|T\|_{\infty}$. Q.E.D.

If $(x, y)$ is an element of $G \cdot b$, there exists a unique pair $\left(x_{0}, c\right) \in S$ $\times C$ such that $x_{0} b=y b$ and $x=x_{0} c$ by Lemma 1.2. Define an isometry $L(x, y)=L^{b}(x, y)$ of $H_{y}$ onto $H_{x}$ by

$$
L(x, y)=L\left(x_{0}, c\right)^{-1} \circ \phi_{x_{0} b, x_{0}}^{b}{ }^{-1} \circ \phi_{y b, y}^{b} .
$$

Then we have the following:

Proposition 1.7. If $b$ is an element of $B$, then $\left(H, L^{b}\right)$ is a representation of $G \cdot b$ in the sense of $[2, p .68$, Definition 1$]$.

Proof. For $(x, y) \in G \cdot b$ with $x_{0} b=y b$ and $x=x_{0} c$, we write $\psi(x$, $\left.y^{\prime}\right)$ for $c$. We have, for $f \in H_{y}$ and $(x, c) \in G$,

$$
\left(L^{b}(x, y) f\right)(x, c)=f(y, \psi(x, y) c) .
$$

Note that $\psi^{-1}(\{c\})$ is a Borel subset of $G \cdot b$ for every $c \in C$. Let $f$ and $g$ be Borel functions on $G$ as in the proof of Proposition 1.5. Then the function $(x, y, c) \mapsto f(y, \psi(x, y) c) g(x, c)$ is Borel on $G \cdot b \times C$. Since we 
have

$$
\left\langle L^{b}(x, y) f_{y}, g_{x}\right\rangle=\int f(y, \psi(x, y) c) g(x, c) d \nu^{x}(x, c),
$$

the function $(x, y) \mapsto\left\langle L^{b}(x, y) f_{y}, g_{x}\right\rangle$ is Borel on $G \cdot b$.

Let $(x, y)$ and $(y, z)$ be elements of $G \cdot b$ such that $x=x_{0} \psi(x, y), y$ $=y_{0} \psi(y, z), x_{0} b=y b$ and $y_{0} b=z b$. We have

$$
x_{0} \psi(y, z)^{-1} b=\left(x_{0} b\right)\left(b^{-1} \psi(y, z)^{-1} b\right)=y \psi(y, z)^{-1} b=z b .
$$

By putting $x_{1}=x_{0} \psi(y, z)^{-1}$, we have $x_{1} b=z b$ and $x_{1} \psi(y, z) \psi(x, y)=x$. It follows that $\psi(x, z)=\psi(y, z) \psi(x, y)$. Similarly we have $\psi(y, x)$ $=\psi(x, y)^{-1}$. The equation $L^{b}\left((y, x)^{-1}(y, z)\right)=L^{b}(y, x)^{-1} L^{b}(y, z)$ follows immediately from the above equations.

Q.E.D.

The following theorem characterizes the von Neumann algebra $\Phi_{b}\left(\operatorname{End}_{\Lambda}(H)\right)$.

Theorem 1.8. Let $b$ be an element of B. For $T \in \operatorname{End}_{A}(H)$, $T$ belongs to $\Phi_{b}\left(\operatorname{End}_{\Lambda}(H)\right)$ if and only if there exists $a G \cdot b$-saturated A-null set $E$ of $S$ such that, for $x, y \in S-E, x_{\widetilde{b}}^{y}$ implies that

$$
L^{b}(x, y) T_{y}=T_{x} L^{b}(x, y) .
$$

Proof. Let $T$ be an element of $\Phi_{b}\left(\operatorname{End}_{\Lambda}(H)\right)$ with $T=\Phi_{b}\left(T^{\prime}\right)\left(T^{\prime}\right.$ $\left.\in \operatorname{End}_{A}(H)\right)$. For $(x, y) \in G \cdot b$ with $x_{0} b=y b$ and $x=x_{0} c$, we have

$$
\begin{aligned}
\phi_{x_{0} b, x_{0}} & { }^{-1} \circ \phi_{y b, y} \circ T_{y} \\
& =\phi_{x_{0} b, x_{0}}{ }^{-1} \circ \phi_{y b, y} \circ \phi_{y b, y}{ }^{-1} \circ T_{y b}^{\prime} \circ \phi_{y b, y} \\
& =\phi_{x_{0} b, x_{0}}{ }^{-1} \circ T^{\prime}{ }_{y b} \circ \phi_{x_{0} b, x_{0}} \circ \phi_{x_{0} b, x_{0}}{ }^{-1} \circ \phi_{y b, y} \\
& =T_{x_{0}} \circ \phi_{x_{0} b, x_{0}}{ }^{-1} \circ \phi_{y b, y} .
\end{aligned}
$$

This implies that $L^{b}(x, y) T_{y}=T_{x} L^{b}(x, y)$ for every $(x, y) \in G \cdot b$.

Since $S$ is a standard space, by [9, Theorem 6.3], there exist a $\Lambda_{\nu}$-null Borel set $N$ of $S$ and a Borel set $S_{0}$ of $S$ such that the restriction $b_{0}$ of $b$ to $S_{0}$ is a one-to-one Borel map of $S_{0}$ onto $S-N$. If we put $\left.N^{\prime}=\cup_{n=0}^{\infty}[N] b^{n}\right) \cup\left(\cup_{n=1}^{\infty}[N] b^{-n}\right)$, then $N^{\prime}$ is a $\Lambda$-null saturated Borel set and $b_{0}$ is a one-to-one Borel map of $S_{0}-N^{\prime}$ onto $S-N^{\prime}$. There- 
fore we may assume that $b_{0}$ is a one-to-one Borel map of $S_{0}$ onto $S$. Note that the inverse map $b_{1}=b_{0}^{-1}$ of $b_{0}$ is Borel. For $x \in S$, the image of $x$ under $b_{1}$ is denoted by $x b_{1}$. Now, suppose that an element $T=\left(T_{x}\right)$ of $\operatorname{End}_{\Lambda}(H)$ and a $\Lambda$-null set $E$ of $S$ satisfy the condition of the theorem. We set

$$
T_{x}^{\prime}=\phi_{x, x b_{1}} \circ T_{x b_{1}} \circ \phi_{x, x b_{1}}{ }^{-1} \quad \text { for } \quad x \in S .
$$

As in the proof of Proposition 1.5, one can prove that $T^{\prime}=\left(T^{\prime}{ }_{x}\right)_{x \in S}$ is a Borel field of operators with respect to $H$. Moreover if $T_{1}$ and $T_{2}$ are intertwining operators of $(H, L)$ which coincide with each other A-almost everywhere, then $T_{1}{ }^{\prime}$ and $T_{2}{ }^{\prime}$ constructed as above coincide with each other $A$-almost everywhere. Let $x$ be an element oí $S-E b$ and $\gamma$ $=(x, c)$ be an element of $G$. If $c^{\prime}$ is an element of $C$ such that $b^{-1} c b$ $=c^{\prime}$, then we have

$$
x b_{1} c b=x b_{1} b c^{\prime}=x c^{\prime},
$$

and there exists $y \in S_{0}$ such that $x b_{1} c b=y b$ i.e. $x c^{\prime} b_{1}=y$. By Lemma 1.4, we have

$$
L\left(x, c^{\prime}\right) \circ \phi_{x c^{\prime}, x b_{1} c}=\phi_{x, x b_{1}} \circ L\left(x b_{1}, c\right) .
$$

This implies that

$$
\begin{gathered}
L\left(x, c^{\prime}\right) \circ \phi_{x c^{\prime}, x b_{1} c^{\circ}} \phi_{y b, x b_{1} c^{-1} \circ \phi_{y b, y}} \\
=\phi_{x, x b_{1}} \circ L^{b}\left(x b_{1}, y\right) .
\end{gathered}
$$

The equation $\phi_{x c^{\prime}, x b_{1} c^{\circ}} \circ \phi_{y b, x b_{1} c}{ }^{-1} \circ \phi_{y b, y}=\phi_{x c^{\prime}, y}$ implies that

$$
L\left(x, c^{\prime}\right) \circ \phi_{x c^{\prime}, y}=\phi_{x, x b_{1}} \circ L^{b}\left(x b_{1}, y\right) .
$$

As $x \notin E b$, we have

$$
\begin{aligned}
& L\left(x, c^{\prime}\right) T^{\prime}{ }_{x c^{\prime}} \\
& =\phi_{x, x b_{1}} \circ L^{b}\left(x b_{1}, y\right) \circ T_{y} \circ \phi_{x c^{\prime}, y}{ }^{-1} \\
& =\phi_{x, x b_{1}} \circ T_{x b_{1}} \circ L^{b}\left(x b_{1}, y\right) \circ \phi_{x c^{\prime}, y}{ }^{-1} \\
& =T^{\prime}{ }_{x} \circ L\left(x, c^{\prime}\right) .
\end{aligned}
$$

Therefore $T^{\prime}$ can be considered as an element of $\operatorname{End}_{A}(H)$ as $E b$ is $\Lambda$-null. Note that $L^{b}\left(x, x b b_{1}\right)=\phi_{x b, x}{ }^{-1} \circ \phi_{x b, x b b_{1}}$. Then, for $x \notin E$, we have 


$$
\begin{aligned}
\Phi_{b}\left(T^{\prime}\right)_{x} & =L^{b}\left(x, x b b_{1}\right) T_{x b b_{1}} L^{b}\left(x, x b b_{1}\right)^{-1} \\
& =T_{x} .
\end{aligned}
$$

Thus $T$ belongs to $\Phi_{b}\left(\operatorname{End}_{\Lambda}(H)\right)$.

Q.E.D.

For the transverse function $\nu$ of counting measures on $G$, we set $m=\int \nu^{x} d \Lambda_{\nu}(x), \nu(H)=\int_{S}^{\oplus} H_{x} d \Lambda_{\nu}(x) \quad$ and, for $T \in \operatorname{End}_{\Lambda}(H), \quad \nu(T)$ $=\int_{S}^{\oplus} T_{x} d \Lambda_{\nu}(x)$. Then $\mathfrak{M}=\nu\left(\operatorname{End}_{\Lambda}(H)\right)$ is a von Neumann algebra on $\nu(H)$ which is isomorphic to $\operatorname{End}_{\Lambda}(H)$ [2, p. 86, Theorem 4]. For $b \in B$, put $\mathfrak{N}_{b}=\nu\left(\Phi_{b}\left(\operatorname{End}_{\Lambda}(H)\right)\right)$. Let $\mathcal{A}$ be a uniformly separable $C^{*}$. subalgebra of $\mathfrak{N}_{b}$ which is weakly dense in $\mathfrak{R}_{b}$. The direct integral decomposition of the identity representation $i$ of $\mathcal{A}$ is denoted by

$$
i=\int_{S}^{\oplus} \widehat{x} d \Lambda_{\nu}(x) \quad[4, \text { Lemma 8.3.1]. }
$$

Note that, for $\nu(T) \in \mathcal{A}$, we have $\hat{x}(\nu(T))=T_{x}$ for $\Lambda_{\nu}$-a.a. $x$. As for the following proposition, compare with $[8,12]$.

Proposition 1.9. In the above situation, there exists a $A$-null set $N$ of $S$ such that, for $x, y \in S-N, \widehat{x}$ and $\hat{y}$ are unitary equivalent if and only if $x \widetilde{b}^{y}$.

Proof. Let $\left\{T_{i}\right\}_{i=1}^{\infty}$ be a uniformly dense subset of $\mathcal{A}$. By Theorem 1.8 , there exists a $\Lambda$-null set $N_{1}$ such that, if $x, y \in S-N_{1}$ and $x \widetilde{b} y$, then $L^{b}(x, y) \hat{y}\left(T_{i}\right)=\widehat{x}\left(T_{i}\right) L^{b}(x, y)$ for every $i$. This means that, for $x, y \in S$ $-N_{1}$ with $x_{\widetilde{b}} y, \hat{x}$ and $\hat{y}$ are unitary equivalent.

Since $S$ is a standard Borel space, we may assume that $S$ has a compact metric topology which is compatible with the Borel structure of $S$. Let $C(S)$ be the $C^{*}$-algebra of all continuous functions on $S$. Let $\left\{g_{i}\right\}_{i=1}^{\infty}$ be a uniformly dense subset of $C(S)$. For every bounded Borel function $g$ on $S$, define an operator $\widehat{g}_{x}$ on $H_{x}$ by

$$
\left(\widehat{g}_{x} f\right)(\gamma)=g(s(\gamma)) f(\gamma) \text { for } f \in H_{x} \quad \text { and } \quad \gamma \in G^{x} .
$$

Then $\widehat{g}=\left(\widehat{g}_{x}\right)_{x \in S}$ belongs to $\operatorname{End}_{\Lambda}(H)$ and we have $\Phi_{b}(\widehat{g})=(b g)^{\wedge}$, where $b g$ is a Borel function on $S$ defined by $(b g)(x)=g(x b)$ for every $x \in S$. 
By [12, Theorem 1.1], we may suppose that $\left\{\nu\left(\left(b g_{i}\right)^{\wedge}\right)\right\}_{i=1}^{\infty}$ is contained in $\mathcal{A}$. There exists a $\Lambda$-null set $N_{2}$ such that, for $x \in S-N_{2}, \widehat{x}\left(\nu\left(\left(b g_{i}\right)^{\wedge}\right)\right)$ $=\left(b g_{i}\right)_{x}$ for every $i$. Suppose that, for $x, y \in S-N_{2}, \hat{x}$ and $\hat{y}$ are unitary equivalent by means of an isometry $V$ of $H_{x}$ onto $H_{y}$. Then we have, for every $i$ and $f \in H_{x}$,

$$
\begin{aligned}
\int g_{i}(x c b)|f(x, c)|^{2} d \nu^{x}(x, c) \\
=\left\langle\left(b g_{i}\right)_{x} f, f\right\rangle \\
=\left\langle V \widehat{x}\left(\nu\left(\left(b g_{i}\right)^{\wedge}\right)\right) f, V f\right\rangle \\
=\left\langle\hat{y}\left(\nu\left(\left(b g_{i}\right)^{\wedge}\right)\right) V f, V f\right\rangle \\
=\left\langle\left(b g_{i}\right)_{y}^{\wedge} V f, V f\right\rangle \\
=\int g_{i}(y c b)|(V f)(y, c)|^{2} d \nu^{y}(y, c) .
\end{aligned}
$$

Since $\left\{g_{i}\right\}$ is uniformly dense in $C(S)$, this implies that, for every Borel set $E$ of $S$ and $f \in H_{x}$,

$$
\begin{aligned}
& \int \chi_{E}(x c b)|f(x, c)|^{2} d \nu^{x}(x, c) \\
&=\int \chi_{E}(y c b)|(V f)(y, c)|^{2} d \nu^{y}(y, c),
\end{aligned}
$$

where $\chi_{E}$ is a characteristic function of $E$. Therefore, for a Borel set $E$ of $S, E b^{-1}$ is $s_{*}\left(\nu^{x}\right)$-null if and only if it is $s_{*}\left(\nu^{y}\right)$-null, where $s_{*}\left(\nu^{x}\right)$ is a measure on $S$ defined by $s_{*}\left(\nu^{x}\right)(E)=\nu^{x}\left(s^{-1}(E)\right)$. As $[x]_{0}$ $=[x] b b^{-1}$ and $s_{*}\left(\nu^{x}\right)\left([x]_{b}\right)>0$, we have $s_{*}\left(\nu^{y}\right)\left([x]_{b}\right)>0$. Since $s_{*}\left(\nu^{y}\right)$ is supported by $[y]$, it follows that $[x]_{b}=[y]_{b}$. If we put $N=N_{1} \cup N_{2}$, then the proposition follows.

Q.E.D.

Theorem 1.10. Let $b_{1}$ and $b_{2}$ be elements of $B$. If there exists an inner automorphism $\alpha$ of $\operatorname{End}_{A}(H)$ such that

$$
\alpha\left(\Phi_{b_{1}}\left(\operatorname{End}_{\Lambda}(H)\right)\right)=\Phi_{b_{2}}\left(\operatorname{End}_{\Lambda}(H)\right),
$$

then $[x]_{b_{1}}=[x]_{b_{2}}$ for $\Lambda$-a.e. $x \in S$.

Proof. Let $U=\left(U_{x}\right)$ be a unitary element of $\operatorname{End}_{\Lambda}(H)$ such that 
$\alpha=\operatorname{Ad} U$. Let $\Re_{i}=\nu\left(\Phi_{b_{i}}\left(\operatorname{End}_{A}(H)\right)\right) \quad(i=1,2)$ and $\mathcal{A}_{1}$ be a uniformly separable $C^{*}$-subalgebra of $\mathfrak{T}_{1}$ which is weakly dense in $\mathfrak{R}_{1}$. We set $\mathscr{A}_{2}=\nu(U) \mathscr{A}_{1} \nu(U)^{*}$. For $x \in S, \hat{x}_{i}$ denotes the representation of $\mathcal{A}_{i}$ on $H_{x}$ defined as above $(i=1,2)$. The isomorphism of $\mathcal{A}_{1}$ onto $\mathcal{A}_{2}$ associated with $\alpha$ is also denoted by $\alpha$. Then we have $U_{x} \widehat{x}_{1} U_{x}{ }^{*}=\widehat{x}_{2} \circ \alpha$ for $\Lambda$-a.a. $x$. It follows that, for $\Lambda$-a.a. $x, y, \hat{x}_{1}$ and $\hat{y}_{1}$ are unitary equivalent by means of an isometry $V$ of $H_{x}$ onto $\mathrm{H}_{y}$ if and only if $\left(U_{y} V U_{x}{ }^{*}\right)$ $\left(\widehat{x}_{2} \circ \alpha\right)\left(U_{y} V U_{x}^{*}\right)^{*}=\hat{y}_{2} \circ \alpha$. The last equation means that $\widehat{x}_{2}$ and $\hat{y}_{2}$ are unitary equivalent. Therefore, by Proposition 1.9, there exists a $\Lambda$-null

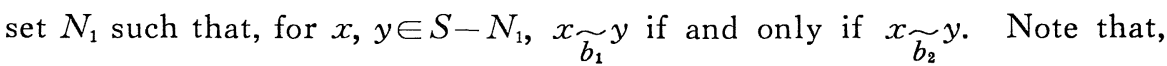
as we have $\left[N_{1}\right]_{b_{1}}=N_{1} b_{1} b_{1}^{-1},\left[N_{1}\right]_{b_{1}}$ is $\Lambda$-null by the condition of Definition 1.3. We set $N=\left[\left[N_{1}\right]_{b_{1}}\right]_{b_{2}}$, which is a $\Lambda$-null set. For $x \in S-N,[x]_{b_{1}}$ is contained in $S-N_{1}$ and $[x]_{b_{2}}$ is contained in $S-N$. From the above argument, we have $[x]_{b_{1}}=[x]_{b_{2}}$.

Q.E.D.

Remark 1.11. Suppose that $(G, \Lambda)$ is ergodic, that is, $(G, \Lambda)$ satisfies one of the equivalent conditions of $[2$, p. 90, Corollary 8]. Then, for every $b \in B$, the relative commutant of $\Phi_{b}\left(\operatorname{End}_{A}(H)\right)$ in $\operatorname{End}_{A}(H)$ is the algebra of scalars.

\section{$\S 2$. Spatially Isomorphism of the Associated von Neumann Algebras}

Let $(A, B, G, A)$ be an action of $B$ on $S$. Throughout this section, we assume that $\Lambda$ is unimodular and that $\mu=\Lambda_{\nu}$ is a probability measure on $S$ for the transverse function $\nu$ of counting measures. If this is the case, $\operatorname{End}_{A}(H)$ is finite. In this section, we show that, if an element $b$ of $B$ satisfies a certain condition, then $\nu\left(\Phi_{b}\left(\operatorname{End}_{A}(H)\right)\right)$ is spatially isomorphic to $\nu\left(\operatorname{End}_{\Lambda}(H)\right) \otimes \mathbf{C}_{k}$, where $\mathbf{C}_{k}$ is the algebra of scalar operators on the $k$-dimensional Hilbert space $\mathfrak{S}_{k}$.

Definition 2. 1. An element $b$ of $B$ is said to be homogeneous of degree $k$ if it satisfies the following conditions;

(i) there exists a Borel partition $\left\{S_{i}\right\}_{i=1}^{k}$ of $S$ such that, for each $i$, the restriction $b_{i}$ of $b$ to $S_{i}$ is a Borel isomorphism of $S_{i}$ onto $S$, 
(ii) if $\mu_{i}$ is the restriction of $\mu$ to $S_{i}$ and $\mu_{i} \cdot\left(b_{i} b_{j}^{-1}\right)$ is a measure on $S_{j}$ defined by $\mu_{i} \cdot\left(b_{i} b_{j}{ }^{-1}\right)(E)=\mu\left(\left\{x \in S_{i} ; x b_{i} b_{j}^{-1} \in E\right\}\right)$ for every Borel set $E$ of $S_{j}$, then $\mu_{i} \cdot\left(b_{i} b_{j}^{-1}\right)=\mu_{j}(i, j=1, \cdots, k)$.

Lemma 2. 2. Let $b$ be homogeneous of degree $k$.

(i) If $\mu \cdot b$ is a measure on $S$ defined by $\mu \cdot b(E)=\mu\left(E b^{-1}\right)$ for every Borel set $E$ of $S$, then $\mu \cdot b$ is equivalent to $\mu$.

(ii) If $\mu \cdot b_{i}^{-1}$ is a measure on $S_{i}$ defined by $\mu \cdot b_{i}{ }^{-1}(E)=\mu\left(E b_{i}\right)$ for every Borel set $E$ of $S_{i}$, then $\mu \cdot b_{i}{ }^{-1}$ is equivalent to $\mu_{i}(i=1, \cdots$, k).

(iii) The equation $\frac{d\left((\mu \cdot b) \cdot b_{i}^{-1}\right)}{d \mu_{i}}=k$ holds $(i=1, \cdots, k)$.

Proof. (i) Note that, as $C$ is a countable discrete group, for a Borel set $E$ of $S, E$ is $\mu$-null if and only if $[E]$ is $\Lambda$-null (c.f. [6, p. 294, Corollary 1]). It follows from the condition of Definition 1.3 that $\mu \cdot b<\mu$. Suppose that $E$ is $\mu \cdot b$-null, that is, $E b^{-1}$ is $\mu$-null. Since $\left[E b^{-1}\right]$ is $\Lambda$-null, $\left[E b^{-1}\right] b$ is also $\Lambda$-null. Since $E$ is contained in $\left[E b^{-1}\right] b$, $E$ is a $\mu$-null set. Thus we have $\mu<\mu \cdot b$. (Note that this proof does not use the homogeneity of $b$ ).

(ii) This can be proved by the same method as that of the proof of (i).

(iii) This follows from straightforward calculation.

Q.E.D.

Let $J_{k}$ be the set of $k$ points $\{1, \cdots, k\}$ and $I_{k}$ be the transitive groupoid $J_{k} \times J_{k}$ on $J_{k}$. For the transverse function $\nu_{k}$ of counting measures on $I_{k}$ and the counting measure $\mu_{k}$ on $J_{k}$, let $\Lambda_{k}$ be the unimodular transverse measure on $I_{k}$ such that $\left(\Lambda_{k}\right)_{\nu_{k}}=\mu_{k}$. Moreover, for the transverse function $\nu \times \nu_{k}$ of counting measures on $G \times I_{k}$, there exists a unique unimodular transverse measure $\Lambda \times \Lambda_{k}$ on $G \times I_{k}$ such that $\left(\Lambda \times \Lambda_{k}\right)_{\nu \times \nu_{k}}$ $=\mu \times \mu_{k}[2$, p. 43, Theorem 3].

Proposition 2.3. Let $b$ be homogeneous of degree $k$. There exists a Borel isomorphism $h$ of the groupoid $G \times I_{k}$ onto the groupoid $G \cdot b$ such that $\mu \sim h_{*}\left(\mu \times \mu_{k}\right)$. Moreover, if $\Lambda^{b}=h\left(\Lambda \times \Lambda_{k}\right)$ is a trans- 
verse measure on $G \cdot b[2, \mathrm{p} .62]$, then $\Lambda_{\nu^{\prime}}^{b}$ is equivalent to $\mu$, where $\nu^{\prime}$ is the transverse function of counting measures on $G \cdot b$.

Proof. We use the notation of Definition 2.1. For $(r,(i, j)) \in G$ $\times I_{k}$, put $h(\gamma, i, j)=\left(r(\gamma) b_{i}^{-1}, s(\gamma) b_{j}^{-1}\right)$, where the image of $x$ under $b_{i}{ }^{-1}$ is denoted by $x b_{i}^{-1}$. It is clear that $h$ is a Borel isomorphism between groupoids $G \times I_{k}$ and $G \cdot b$. For a Borel set $E$ of $S$, we have

$$
h_{*}\left(\mu \times \mu_{k}\right)(E)=\sum_{i=1}^{k} \mu \cdot b_{i}^{-1}\left(E \cap S_{i}\right) .
$$

It follows from Lemma 2.2 that $h_{*}\left(\mu \times \mu_{k}\right)$ is equivalent to $\mu$.

We use the notation in $\left[2\right.$, p. 62]. For $\nu^{\prime \prime} \in \mathcal{E}^{+}(G \cdot b)$, we have, by the definition,

$$
\Lambda^{b}\left(\nu^{\prime \prime}\right)=\int h^{*}\left(L^{\nu^{\prime \prime}}\right) d\left(\Lambda \times \Lambda_{k}\right) .
$$

Note that, for a Borel set $E$ of $S \times J_{k}$,

$$
\Lambda_{\nu^{\prime}}^{b}(E)=\int h^{*}\left(L^{\left(\chi_{\left.E^{\circ} s\right) \nu^{\prime}}\right.}\right) d\left(\Lambda \times \Lambda_{k}\right)=0
$$

if and only if

$$
\left\{(x, i) \in S \times J_{k} ;\left(\left(\chi_{E} \circ S\right) \nu^{\prime}\right)^{h(x, i)} \neq 0\right\} \text { is } \Lambda \times \Lambda_{k} \text {-null }
$$

[2, p. 56, Lemma 5]. On the other hand, we have

$$
\left\{(x, i) \in S \times J_{k} ;\left(\left(\chi_{E} \circ s\right) \nu^{\prime}\right)^{h(x, i)} \neq 0\right\}=\mathrm{h}^{-1}\left([E]_{b}\right) .
$$

Therefore, by the formula $\mu \sim h_{*}\left(\mu \times \mu_{k}\right)$, we know that $\Lambda_{\nu}^{b},(E)=0$ if and only if $[E]_{b}$ is $\mu$-null. Since we have $[E]_{b}=[E b] b^{-1},[E]_{b}$ is a $\mu$-null set if and only if $E b$ is a $\mu$-null set. We have

$$
\begin{array}{cl}
\mu(E b)=0 \quad & \text { iff } \quad \mu \cdot b_{i}^{-1}\left(E \cap S_{i}\right)=0 \quad(i=1, \cdots, k) \\
& \text { iff } \quad \mu\left(E \cap S_{i}\right)=0 \quad(i=1, \cdots, k) \\
& \text { iff } \mu(E)=0 .
\end{array}
$$

It follows that $\Lambda_{\nu^{\prime}}^{b}(E)=0$ if and only if $\mu(E)=0$.

Q.E.D.

Remark 2. 4. The representation $\left(H, L^{b}\right)$ of $G \cdot b$ defined in Section 1 is a square integrable representation [2, p. 80 , Definition]. If $b$ is homogeneous, then $\nu^{\prime}\left(\operatorname{End}_{\Lambda^{b}}(H)\right)$ and $\nu\left(\Phi_{b}\left(\operatorname{End}_{\Lambda}(H)\right)\right)$ are spatially isomor- 
phic, where $\operatorname{End}_{4^{b}}(H)$ is the von Neumann algebra associated with $\left(H, L^{b}\right)$.

The measure $\int \nu^{x} d \mu(x)$ on $G$ is denoted by $m$. We set $\mathfrak{M}=$ $\nu\left(\operatorname{End}_{A}(H)\right), \mathfrak{N}_{b}=\nu\left(\Phi_{b}\left(\operatorname{End}_{A}(H)\right)\right)$ and $\mathfrak{S}=\nu(H)$. The Hilbert space $\mathfrak{E}$ can be identified with $L^{2}(G, m)$. Define a partial isometry $U=U_{b}$ on $\mathfrak{S}$ by

$$
U(f)(x, c)=\left(\frac{d(\mu \cdot b)}{d \mu}\right)^{-1 / 2}(x b) f\left(x b, b^{-1} c b\right)
$$

for $f \in \mathfrak{H}$ and $(x, c) \in G$.

Lemma 2.5. Let $b$ be homogeneous of degree $k$ and $e$ be the final projection of $U_{b}$.

(i) The space eSc consists of all elements $f=\left(f_{x}\right)$ of $\mathfrak{S}$ which satisfy the following; there exists a saturated null set $N=N_{f}$ of $S$ such that $f_{x}=L^{b}(x, y) f_{y}$ if $x b=y b$ and $x, y \in S-N$.

(ii) The projection e belongs to $\mathfrak{N}_{b}^{\prime}$.

Proof. (i) Let $\mathfrak{S}_{0}$ be the space consisting of all elements which satisfy the condition of (i). We write $U$ for $U_{b}$. If $x b=y b$, then we have, for $f \in \mathfrak{S}$,

$$
\begin{aligned}
\left(L^{b}(x, y)(U f)_{y}\right)(x, c) & =(U f)_{y}(y, c) \\
& =(U f)_{x}(x, c) .
\end{aligned}
$$

Hence $e \mathfrak{S}$ is contained in $\mathfrak{W}_{0}$. Conversely, let $f$ be an element in $\mathfrak{H}_{0}$. For $x, y \in S-N_{f}$ with $x b=y b$, we have $f(x, c)=f(y, c)$ for all $c \in C$. Fix an integer $i$ with $1 \leq i \leq k$ and define an element $g$ of $\mathfrak{S}$ by

$$
g(x, c)=k^{1 / 2}\left(\frac{d\left(\mu \cdot b_{i}^{-1}\right)}{d \mu_{i}}\right)^{-1 / 2}\left(x b_{i}^{-1}\right) f\left(x b_{i}^{-1}, b c b^{-1}\right)
$$

$((x, c) \in G)$. Then we have, for $(x, c) \in G$.

$$
\begin{aligned}
& U(g)(x, c) \\
& \quad=k^{1 / 2}\left(\frac{d(\mu \cdot b)}{d \mu}\right)^{-1 / 2}(x b)\left(\frac{d\left(\mu \cdot b_{i}{ }^{-1}\right)}{d \mu_{i}}\right)^{-1 / 2}\left(x b b_{i}{ }^{-1}\right) f\left(x b b_{i}{ }^{-1}, c\right) .
\end{aligned}
$$

On the other hand, we have, for $\mu$-a.a. $x \in S$, 


$$
\begin{aligned}
\frac{d(\mu \cdot b)}{d \mu} & (x b) \frac{d\left(\mu \cdot b_{i}^{-1}\right)}{d \mu_{i}}\left(x b b_{i}^{-1}\right) \\
= & \frac{d\left((\mu \cdot b) \cdot b_{i}^{-1}\right)}{d \mu_{i}}\left(x b b_{i}{ }^{-1}\right)=k .
\end{aligned}
$$

It follows that $U(g)(x, c)=f\left(x b b_{i}{ }^{-1}, c\right)$ for $\mu$-a.a. $x \in S$ and all $c \in C$. Since we have $f\left(x b b_{i}{ }^{-1}, c\right)=f(x, c)$ for $x \notin N_{f} \cup N_{f} b_{i} b^{-1}$, we have $U(g)$ $=f$. Hence $\mathfrak{S}_{0}$ is contained in $e \mathfrak{g}$.

(ii) Let $T$ be an element of $\mathfrak{N}_{b}$ and $f$ be an element of $e \mathfrak{S}$. For $x, y \in S-N_{f}$ with $x b=y b$. we have

$$
L^{b}(x, y) T_{y} f_{y}=T_{x} L^{b}(x, y) f_{y}=T_{x} f_{x} .
$$

Therefore $T f$ belongs to $e \mathfrak{S}$ and $e$ is an element of $\mathfrak{Y l}_{b}^{\prime}$.

Q.E.D.

Lemma 2. 6. Let $e$ be as in Lemma 2.5 and $\mathfrak{S}_{k}$ be a k-dimensional Hilbert space whose complete orthonormal system is $\left\{\delta_{i}\right\}_{i=1}^{k}$. For $f \otimes \delta_{i} \in e \mathfrak{S} \otimes \mathfrak{C}_{k}$, define an element $\psi\left(f \otimes \delta_{i}\right)$ of $\mathfrak{S}$ by the following; $\psi\left(f \otimes \delta_{i}\right)_{x}$ is $k^{1 / 2} f_{x}$ if $x \in S_{i}$ and is 0 if $x \notin S_{i}$. Then $\psi$ can be

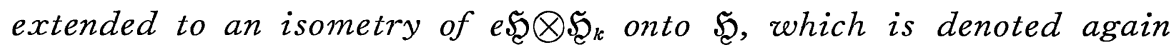
by $\psi$. Moreover the von Neumann algebras $\left(\mathfrak{R}_{b}\right)_{e} \otimes C_{k}$ and $\mathfrak{N}_{b}$ are spatially isomorphic by means of $\psi$, where $\boldsymbol{C}_{k}$ is the algebra of scalar operators on $\mathfrak{S}_{k}$.

Proof. For $f \in e \mathfrak{S}$, we have

$$
\begin{aligned}
& \left\|\psi\left(f \otimes \delta_{i}\right)\right\|^{2} \\
& =\int_{S_{i}}\left\|\psi\left(f \otimes \delta_{i}\right)_{x}\right\|^{2} d \mu_{i}(x) \\
& \quad=k \int_{S_{i}}\left\|f_{x}\right\|^{2} d \mu_{i}(x) \\
& =\sum_{j=1}^{k} \int_{S_{i}}\left\|L^{b}\left(x b_{i} b_{j}^{-1}, x\right) f_{x}\right\|^{2} d \mu_{i}(x) \\
& =\sum_{j=1}^{k} \int_{S_{i}}\left\|f_{x b_{i} b_{j^{-1}}}\right\|^{2} d \mu_{i}(x) \\
& =\sum_{j=1}^{k} \int_{S_{j}}\left\|f_{x}\right\|^{2} d\left(\mu_{i} \cdot\left(b_{i} b_{j}^{-1}\right)\right)(x)
\end{aligned}
$$




$$
=\|f\|^{2} \text {. }
$$

It follows that $\psi$ can be extended to an isometry of $e \mathfrak{S} \otimes \mathfrak{H}_{k}$ into $\mathfrak{H}$. Let $f_{0}$ be an element of $\mathfrak{H}$ such that $\left\langle f_{0}, f\right\rangle=0$ for all $f \in \psi\left(e \mathfrak{E} \otimes \mathbb{C}_{k}\right)$. For $f \in \mathfrak{E}$, define an element $f^{i}$ of $e \mathfrak{E}$ by $f^{i}(x, c)=f\left(x b_{j} b_{i}{ }^{-1}, c\right)$ for $x \in S_{j}$ and $c \in \mathrm{C}(j=1, \cdots, k)$. Then we have

$$
\begin{aligned}
k^{1 / 2} \int_{S_{i}}\left\langle f_{0 x}, f_{x}\right\rangle d \mu(x) & =\left\langle f_{0}, \psi\left(f^{i} \otimes \delta_{i}\right)\right\rangle \\
& =0 .
\end{aligned}
$$

Since $f$ and $i$ are arbitrary, we have $f_{0}=0$. Hence $\psi$ is onto.

Let $T$ be an element of $\left(\mathfrak{N}_{b}\right)_{e}$. There exists an element $T^{\prime}$ of $\mathfrak{N}_{b}$ such that $T^{\prime}{ }_{e}=T$. For $f \otimes \delta_{i} \in e \mathfrak{S}_{\mathcal{C}} \otimes \mathfrak{S}_{k}$, we have $\psi\left((T \otimes I)\left(f \otimes \delta_{i}\right)\right)_{x}$ $=\left(T^{\prime} \psi\left(f \otimes \delta_{i}\right)\right)_{x}$ for a.a. $x \in S$. It follows that $\psi(T \otimes I)=T^{\prime} \psi$. If $T^{\prime}$ and $T^{\prime \prime}$ are elements of $\mathfrak{N}_{b}$ such that $T^{\prime}{ }_{e}=T^{\prime \prime}{ }_{e}=T$, then we have $T^{\prime}$ $=\psi(T \otimes I) \psi^{-1}=T^{\prime \prime}$. This means that the map $T^{\prime} \mapsto T_{e}^{\prime}$ is an isomorphism of $\mathfrak{N}_{b}$ onto $\left(\mathfrak{N}_{b}\right)_{e}$. Therefore the map $T \otimes I \mapsto \psi(T \otimes I) \psi^{-1}$ is an isomorphism of $\left(\mathfrak{N}_{b}\right)_{e} \otimes \mathbf{C}_{k}$ onto $\mathfrak{N}_{b}$.

Q.E.D.

From the above proof, it is also shown that the central support of $e$ is $I$.

Lemma 2. 7. Let $U$ and $e$ be as in Lemma 2.5. The von Neumann algebras $\left(\mathfrak{N}_{b}\right)_{e}$ and $\mathfrak{M}$ are spatially isomorphic by means of $U$.

Proof. For $T \in\left(\mathfrak{N}_{b}\right)_{e}$, there exists a unique element $T^{\prime}$ of $\mathfrak{M}$ such that $\Phi_{b}\left(T^{\prime}\right)_{e}=T$. As we have, for $f \in \mathfrak{S}$,

$$
U(f)_{x}=\left(\frac{d(\mu \cdot b)}{d \mu}\right)^{1 / 2}(x b) \phi_{x b, x}{ }^{-1}\left(f_{x b}\right),
$$

we have

$$
\begin{aligned}
(T U f)_{x} & =\Phi_{b}\left(T^{\prime}\right)_{x} U(f)_{x} \\
& =\left(\frac{d(\mu \cdot b)}{d \mu}-\right)^{1 / 2}(x b) \phi_{x b, x}{ }^{-1}\left(T_{x b}^{\prime} f_{x b}\right) \\
& =U\left(T^{\prime} f\right)_{x} .
\end{aligned}
$$

It follows that $T^{\prime}=U^{*} T U$.

Q.E.D. 
From the above lemmas, we get the following theorem.

Theorem 2.8. If $b$ is homogeneous of degree $k$, then $\mathfrak{M} \otimes \mathbf{C}_{k}$ and $\mathfrak{N}_{b}$ are spatially isomorphic.

Corollary 2.9. Suppose that $(G, \Lambda)$ is ergodic. Let $b_{i}$ be homogeneous of degree $k_{i}(i=1,2)$. If $k_{1} \neq k_{2}$, then there are no automorphisms of $\operatorname{End}_{\Lambda}(H)$ which send $\Phi_{b_{1}}\left(\operatorname{End}_{\Lambda}(H)\right)$ onto $\Phi_{b_{2}}\left(\operatorname{End}_{\Lambda}(H)\right)$.

Proof. By the ergodicity of $(G, \Lambda), \mathfrak{M}$ and $\mathfrak{N}_{b_{i}}(i=1,2)$ are factors. Since $\mathfrak{M}$ is standard, the coupling constant of $\mathfrak{N}_{b_{i}}$ is $k_{i} \quad(i=1,2)$ [11, Corollary 7.22]. It follows that $\mathfrak{N}_{b_{1}}$ and $\mathfrak{N}_{b_{z}}$ cannot be spatially isomorphic if $k_{1} \neq k_{2}$ [11, Theorem 8.3]. On the other hand, any automorphism of $\mathfrak{M}$ is spatial [3, p. 268, Corollary]. Therefore there are no automorphisms of $\mathfrak{M}$ which send $\mathfrak{N}_{b_{1}}$ onto $\mathfrak{N}_{b_{2}}$.

Q.E.D.

\section{§ 3. An Example}

In this section, we apply the results of the previous sections to the action of the special linear group $S L(n, \mathbf{Z})$ of degree $n$ on the $n$-dimensional torus $\mathbf{T}^{n}(n \geq 2)$. Let $A$ be the normalizer of $S L(n, \mathbf{Z})$ in the general linear group $G L(n, \mathbf{Q})$, and $B$ be the semigroup consisting of all elements of $A$ whose coefficients are integers. Note that $B$ contains the elements of the form $k I(k \in \mathbf{Z}-\{0\})$, where $I$ is the unit matrix. We set $C=S L(n, \mathbf{Z})$ and $S=\mathbf{T}^{n}$. The action of $b=\left(b_{i j}\right) \in B$ on $x=$ $\left(x_{1}, \cdots, x_{n}\right) \in S$ is defined by

$$
x b=\left(\sum_{j=1}^{n} b_{j 1} x_{j}, \cdots, \sum_{j=1}^{n} b_{j n} x_{j}\right) \quad\left(\bmod \mathbf{Z}^{n}\right) .
$$

Let $G_{n}$ be the groupoid $S \times C$ associated with the above action and $\Lambda$ be the transverse measure on $G_{n}$ such that $\Lambda_{\nu}$ is the Lebesgue measure $\mu$ on $S$ for the transverse function $\nu$ of counting measures. Note that $\Lambda$ is unimodular and ergodic. Since the action of $C$ on $S$ is essentially free (c.f. [13, Proof of Corollary 4.6.]), $(A, B, G, \Lambda)$ can be considered as an action of $B$ on $S$ (Definition 1.3). Let $\mathfrak{M}_{n}$ be the von Neumann algebra $\nu\left(\operatorname{End}_{\Lambda}(H)\right)$ associated with the left regular representation 
$\left(H, L^{\nu}\right)$ of $G_{n}$. Then $\mathfrak{M}_{n}$ is a factor of type $\mathrm{II}_{1}$, which coincides with the factor obtained by the group-measure space construction. For $k \in \mathbb{Z}-\{0\}$, the element $k I$ of $B$ is homogeneous of degree $|k|^{n}$. We set $\mathfrak{N}_{k}^{n}=\nu\left(\Phi_{k I}\right.$ $\left.\left(\operatorname{End}_{\Lambda}(H)\right)\right)$. The following theorem summarizes properties of $\mathfrak{N}_{k}^{n}$ which are obtained from the results of the previous sections.

Theorem 3.1. (i) The subfactor $\mathfrak{N}_{k}^{n}$ of $\mathfrak{M}_{n}$ is spatially isomorphic to $\mathfrak{M}_{n} \otimes \mathbf{C}_{|k|^{n}} \quad(k \in \mathbf{Z}-\{0\})$.

(ii) Elements of the family $\left\{\mathfrak{N}_{k}^{n}\right\}_{k \in \mathbf{N}}$ are not conjugate with each other by any automorphism of $\mathfrak{M i}_{n}$.

(iii) For $k, l \in \mathbf{N}, k$ is a divisor of $l$ if and only if $\mathfrak{\Re}_{k}^{n} \supset \mathfrak{N}_{l}^{n}$.

(iv) For $p, q \in \mathbf{N}-\{1\}, p$ and $q$ are relatively prime if and only if $\mathfrak{N}_{p}^{n} \cap \mathfrak{N}_{q}^{n}=\mathfrak{N}_{p q}^{n}$.

Proof. The statements of (i) and (ii) are clear by Theorem 2.8 and Corollary 2.9. We will show the statements of (iii) and (iv). For $k \in \mathbb{N}$, we write $k$ instead of $k I$.

(iii) By Theorem 1.8, if $k$ is a divisor of $l$, then $\mathfrak{N}_{k}^{n} \supset \mathfrak{N}_{l}^{n}$. Conversely, suppose that $\mathfrak{R}_{k}^{n} \supset \Re_{l}^{n}$. Let $\mathcal{A}$ be a uniformly separable $C^{*}$-subalgebra of $\mathfrak{N}_{k}^{n}$ such that $\mathcal{A}$ is weakly dense in $\mathfrak{N}_{k}^{n}$ and such that $\mathcal{A}_{1}=$ $\mathcal{A} \cap \mathfrak{N}_{l}^{n}$ is weakly dense in $\mathfrak{N}_{l}^{n}$. For $x \in S$, let $\widehat{x}$ (resp. $\widehat{x}_{1}$ ) be the representation of $\mathcal{A}$ (resp. $\mathscr{A}_{1}$ ) on $H_{x}$ defined as in Section 1 . Note that, for $\mu$-a.a. $x, \widehat{x}_{1}$ is the restriction of $\widehat{x}$ to $\mathcal{A}_{1}$. By Proposition 1.9 , for $\mu$-a.a. $x, y$, if $x \widetilde{k} y$, then $\widehat{x}_{1}$ and $\hat{y}_{1}$ are unitary equivalent and so we have $x_{\widetilde{l}} y$. It follows that $[x]_{k} \subset[x]_{l}$ for $\mu$-a.a. $x$. For such an $x$, define an element $y$ of $S$ by

$$
y=x+(1 / k, \cdots, 1 / k) \quad\left(\bmod \mathbb{Z}^{n}\right) .
$$

Since $y$ belongs to $[x]_{l}$, we have $x l=y l c\left(\bmod \mathbb{Z}^{n}\right)$ for some $c \in C$. We may suppose that the stability subgroup of $x$ in $C$ is trivial. Then $c$ is a unit element and we have $x l=y l\left(\bmod \mathbf{Z}^{n}\right)$. Thus we have $l / k$ $=1(\bmod \mathbb{Z})$.

(iv) As we have $\Phi_{p} \circ \Phi_{q}=\Phi_{p q}$ (Lemma 1.6, (i)), $\mathfrak{N}_{p q}^{n}$ is contained in $\mathfrak{N}_{p}^{n} \cap \mathfrak{N}_{q}^{n}$. Suppose that $p$ and $q$ are relatively prime. Then there exist integers $t$ and $u$ such that $p t+q u=1$. For $x, y \in S$ with $x \widetilde{p q} y$, there 
exists $y_{0} \in S$ such that $x p q=y_{0} p q$ and $y_{0} \sim y$. For some $m_{i} \in \mathbf{Z}(i=1, \cdots$, $n$ ), we have

$$
\begin{aligned}
y_{0} & =x+\left(m_{1} / p q, \cdots, m_{n} / p q\right) \quad\left(\bmod \mathbf{Z}^{n}\right) \\
& =x+\left(m_{1} u / p, \cdots, m_{n} u / p\right)+\left(m_{1} t / q, \cdots, m_{n} t / q\right) \quad\left(\bmod \mathbf{Z}^{n}\right) .
\end{aligned}
$$

If we set $z=x+\left(m_{1} u / p, \cdots, m_{n} u / p\right)\left(\bmod \mathbf{Z}^{n}\right)$, then we have $x_{\widetilde{p}} z, z_{\widetilde{q}} y_{0}$ and $z \widetilde{q}_{q} y$. By calculation, we have $L^{p q}(x, y)=L^{p}(x, z) L^{q}(z, y)$. For $T \in \mathfrak{N}_{p}^{n} \cap \Re_{q}^{n}$ and $\mu$-a.a. $x, y \in S$ with $x \widetilde{p q} y$, we have

$$
\begin{aligned}
L^{p q}(x, y) T_{y} & =L^{p}(x, z) L^{q}(z, y) T_{y} \\
& =L^{p}(x, z) T_{z} L^{q}(z, y) \\
& =T_{x} L^{p q}(x, y) .
\end{aligned}
$$

It follows from Theorem 1.8 that $T$ is an element of $\Re_{p q}^{n}$. Suppose now that $p$ and $q$ are not relatively prime. Let $k$ be the least common multiple of $p$ and $q$. It follows from (iii) that $\mathfrak{N}_{k}^{n} \subset \mathfrak{N}_{p}^{n} \cap \mathfrak{N}_{q}^{n}$ and $\mathfrak{N}_{p q}^{n} \subset \mathfrak{N}_{k}^{n}$. As $k \neq p q, \mathfrak{N}_{k}^{n}$ does not coincide with $\mathfrak{N}_{p q}^{n}$. Hence we have $\mathfrak{N}_{p}^{n} \cap \mathfrak{N}_{q}^{n} \neq \mathfrak{N}_{p q}^{n}$.

Q.E.D.

For $k \in \mathbf{N}$, we set $\mathfrak{N}_{k \infty}^{n}=\bigcap_{i=1}^{\infty} \mathfrak{N}_{k i}^{n}$ and $G_{n} \cdot\left(k^{\infty}\right)=\cup_{i=1}^{\infty} G_{n} \cdot\left(k^{i}\right)$. It is clear that $\mathfrak{N}_{k \infty}^{n}$ is a finite von Neumann algebra and that $G_{n} \cdot\left(k^{\infty}\right)$ is a measurable groupoid whose Borel structure is standard. For $(x, y) \in G_{n}$ $\cdot\left(k^{\infty}\right),(x, y)$ belongs to $G_{n} \cdot\left(k^{i}\right)$ for some $i$. Define an isometry $L^{k^{\infty}}(x$, $y$ ) of $H_{y}$ onto $H_{x}$ by $L^{k \infty}(x, y)=L^{k i}(x, y)$. If $(x, y) \in G_{n} \cdot\left(k^{j}\right)$, then $L^{k j}(x, y)=L^{k^{i}}(x, y)$. Therefore $L^{k \infty}$ is well-defined. By Proposition 1.7, $\left(H, L^{k^{\infty}}\right)$ is a representation of $G_{n} \cdot\left(k^{\infty}\right)$. For $T \in \mathfrak{M}_{n}, T$ is in $\mathfrak{N}_{k \infty}^{n}$ if and only if there exists a $\mu$-conull set $E$ of $S=\left(G_{n} \cdot\left(k^{\infty}\right)\right)^{(0)}$ such that $L^{k^{\infty}}(x, y) T_{y}=T_{x} L^{k^{\infty}}(x, y)$ for $(x, y) \in G_{n} \cdot\left(k^{\infty}\right) \mid E$ (c.f. Theorem 1.8). Let $R_{k}$ be an equivalence relation on $S$ defined by the following; $(x, y)$ $\in R_{k}$ if and only if there exist $i \in \mathbf{N}$ and $m_{j} \in \mathbf{Z}(j=1, \cdots, n)$ such that

$$
y=x+\left(m_{1} / k^{i}, \cdots, m_{n} / k^{i}\right) \quad\left(\bmod \mathbf{Z}^{n}\right) .
$$

For $k \geq 2, R_{k}$ is hyperfinite (c.f. [6, Proposition 4.1]) and is an ergodic groupoid of type $\mathrm{II}_{1}$ with respect to the measure $\mu$. For every $(x, y)$ $\in G_{n} \cdot\left(k^{\infty}\right)$, there exists $z \in S$ such that $(x, z) \in R_{k}$ and $(z, y) \in G_{n}$. 
Theorem 3.2. If $k$ is a natural number with $k \geq 2$, then $\mathfrak{N}_{k_{\infty}}^{n}$ and $\lambda(C) " \otimes \mathbf{C}_{S}$ are spatially isomorphic, where $\lambda$ is the right regular representation of $C$ and $\mathbb{C}_{S}$ is the algebra of scalar operators on $L^{2}(S, \mu)$.

Proof. We set $\mathfrak{g}=\nu(H)$ and $\mathfrak{S}_{0}=L^{2}(S, \mu)$. For $c \in C$, define a unitary operator $U_{c} \in \mathfrak{M}_{n}$ by $\left(U_{c} f\right)\left(x, c^{\prime}\right)=f\left(x, c^{\prime} c\right)$ for $f \in \mathfrak{S}$ and $\left(x, c^{\prime}\right)$ $\in G_{n}$. Let $L^{\infty}(S)$ be the set of all bounded Borel functions on $S$. For $\alpha \in L^{\infty}(S)$, define $\hat{\alpha} \in \mathfrak{M}_{n}$ by $(\hat{\alpha} f)(x, c)=\alpha(x c) f(x, c)$ for $f \in \mathfrak{H}$ and $(x, c) \in G_{n}$. For $b \in B, b \cdot \alpha$ denotes an element of $L^{\infty}(S)$ defined by $(b \cdot \alpha)(x)=\alpha(x b) \quad(x \in S)$. Then we have $U_{c} \hat{\alpha} U_{c}{ }^{*}=(c \cdot \alpha)^{\wedge}$ for $c \in C$ and $\alpha \in L^{\infty}(S)$. If we set $\mathfrak{U}=\left\{\hat{\alpha} ; \alpha \in L^{\infty}(S)\right\}$, then $\mathfrak{M}_{n}$ is generated by $\mathfrak{A}$ and $\left\{U_{c} ; c \in C\right\}$. Since $k=k I$ commutes with every element of $C$, it follows from straightforward calculation that $\Phi_{k}(\hat{\alpha})=(k \cdot \alpha)^{\wedge}$ and $\Phi_{k}\left(U_{c}\right)=U_{c}$ for every $\hat{\alpha} \in \mathfrak{A}$ and $c \in C$.

As $\mathfrak{S}$ is isomorphic to $\mathfrak{S}_{0} \otimes l^{2}(C)$, we may represent elements of $\mathfrak{M N}_{n}$ by matrices. For $c \in C$, let $\delta_{c}$ be an element of $l^{2}(C)$ defined by $\delta_{c}(c)=1$ and $\delta_{c}\left(c^{\prime}\right)=0$ if $c^{\prime} \neq c$, and $\mathfrak{S}_{c}$ be the one-dimensional subspace of $l^{2}(C)$ generated by $\delta_{c}$. We define an isometry $v_{c}$ of $\mathfrak{S}_{0}$ onto $\mathfrak{S}_{0} \otimes \mathfrak{S}_{\mathfrak{c}}$ by $\boldsymbol{v}_{c}(f)$ $=f \otimes \delta_{c}$ for $f \in \mathfrak{S}_{0} . \quad$ For $T \in \mathfrak{M}_{n}$, we set $T_{t, u}=v_{t}^{-1} T v_{u} \quad(t, u \in C)$ and identify $T$ with the matrix $\left(T_{t, u}\right)_{t, u \in C}$. Then $U_{c}(c \in C)$ is represented by the following; $\left(U_{c}\right)_{t, u}=I$ if $t^{-1} u=c$ and $\left(U_{c}\right)_{t, u}=0$ if $t^{-1} u \neq c$, where $I$ is the identity operator on $\mathfrak{S}_{0}$, and $\hat{\alpha}\left(\alpha \in L^{\infty}(S)\right)$ is represented by the following; $(\hat{\alpha})_{t, u}=t \cdot \alpha$ if $t=u$ and $(\hat{\alpha})_{t, u}=0$ if $t \neq u$, where $t \cdot \alpha$ is considered as a multiplication operator on $\mathfrak{S}_{\jmath}$. Therefore $\Phi_{k}\left(\hat{\alpha} U_{c}\right)$ is represented by the following; $\Phi_{k}\left(\hat{\alpha} U_{c}\right)_{t, u}=t \cdot(k \cdot \alpha)$ if $t^{-1} u=c$ and $\Phi_{k}\left(\hat{\alpha} U_{c}\right)_{t, u}=0$ if $t^{-1} u \neq c$. Let $\mathfrak{M}_{0}$ be a ${ }^{*}$-subalgebra of $\mathfrak{M}_{n}$ consisting of all elements of the form $\hat{\alpha}_{1} U_{c_{1}}+\cdots+\hat{\alpha}_{m} U_{c_{m}}$ for $\alpha_{i} \in L^{\infty}(S)$ and $c_{i} \in C$ (i $=1, \cdots, m ; m=1,2, \cdots)$. As $\mathfrak{M}_{0}$ is weakly dense in $\mathfrak{M}_{n}, \Phi_{k}\left(\mathfrak{M}_{0}\right)$ is weakly dense in $\mathfrak{N}_{k}^{n}$. By the same reasoning as in [3, p. 131], for every $T$ $=\left(T_{t, u}\right) \in \mathfrak{N}_{k}^{n}$, there exists $\alpha_{c} \in L^{\infty}(S) \quad(c \in C)$ such that $T_{t, u}=t \cdot\left(k \cdot \alpha_{t-1 u}\right)$ for every $t, u \in C$. Note that, for $x, y \in S$, if $y=x+\left(m_{1} / k, \cdots, m_{n} / k\right)$ $\left(\bmod \mathbf{Z}^{n}\right)$ for some $m_{i} \in \mathbf{Z}(i=1, \cdots, n)$, then we have $k \cdot \alpha(x)=k \cdot \alpha(y)$ for $\alpha \in L^{\infty}(S)$. Let $T=\left(T_{t, u}\right)$ be an element of $\mathfrak{N}_{k \infty}^{n}$ such that $T_{t, u}=t$ - $\alpha_{t-1 u}$ for $t, u \in C\left(\alpha_{c} \in L^{\infty}(S), c \in C\right)$. It follows from the above argument that there exists a $\mu$-conull set $S_{0}$ of $S$ such that $\alpha_{c}(x)=\alpha_{c}(y)$ 
for every $(x, y) \in R_{k} \mid S_{0}$ and $c \in C$. Since $R_{k}$ is ergodic with respect to the measure $\mu, \alpha_{c}$ is constant $\mu$-a.e. for all $c \in C$. Therefore there exists a von Neumann algebra $\mathfrak{N}_{0}$ on $l^{2}(C)$ such that $\mathfrak{N}_{k \infty}^{n}=\mathbf{C}_{s} \otimes \mathfrak{N}_{0}$. Let $T^{0}$ be an element of $\mathfrak{N}_{0}$. We shall represent $T^{0}$ by a matrix with respect to the base $\left\{\delta_{c}\right\}_{c \in C}$ of $l^{2}(C)$. There exists $w_{c} \in \mathbf{C}(c \in C)$ such that $T^{0}{ }_{t, u}$ $=w_{t-1 u}$ for $t, u \in C$. Let $\rho$ be the left regular representation of $C$, which is represented by the following; $\rho(c)_{t, u}=1$ if $t \iota^{-1}=c$ and $\rho(c)_{t, u}=0$ if $t u^{-1} \neq c$. Then we have

$$
\begin{aligned}
\left(\rho(c) T^{0}\right)_{t, u} & =w_{t-1 c u} \\
& =\left(T^{0} \rho(c)\right)_{t, u} .
\end{aligned}
$$

Hence $T^{0}$ belongs to $\rho(C)^{\prime}$. As we have $\rho(C)^{\prime}=\lambda(C)^{\prime \prime}[4,13.10]$, $\mathfrak{N}_{0}$ is contained in $\lambda(C)^{\prime \prime}$. On the other hand, as we have $U_{c} \in \mathfrak{N}_{k \infty}^{n}$ and $U_{c}=I \otimes \lambda(c), \lambda(C)$ " is contained in $\mathfrak{N}_{0}$. Thus we have $\mathfrak{N}_{k \infty}^{n}=\mathbf{C}_{s} \otimes \lambda(C) "$.

Q.E.D.

The following corollary is an immediate consequence of the above proof.

Corollary 3.3. (i) The fixed point algebra of $\Phi_{k}$ is $\mathfrak{N}_{k \infty}^{n}$, that is,

$$
\mathfrak{R}_{k \infty}^{n}=\left\{T \in \mathfrak{M}_{n} ; \Phi_{k}(T)=T\right\} .
$$

(ii) If $k$ and $l$ are natural numbers with $k, l \geq 2$, then $\mathfrak{N}_{k \infty}^{n}$ coincides with $\mathfrak{R}_{l^{\infty}}^{n}$.

\section{References}

[1] Auslander, L. and Moore, C. C., Unitary representations of solvable Lie groups, Mem. Amer. Math. Soc., 62 (1966).

[2] Connes, A., Sur la théorie non commutative de l'intégration, Springer Lecture Notes in Math., 725 (1979), 19-143.

[3] Dixmier, J., Les algèbres d'opérateurs dans l'espace Hilbertien, Gauthier-Villars, Paris, 1969.

[4] - C $C^{*}$-algebras, North-Holland, Amsterdam, 1977.

[5] Feldman, J., Hahn, P. and Moore, C. C., Orbit structure and countable sections for actions of continuous groups, Advances in Math., 28 (1978), 186-230.

[6] Feldman, J. and Moore, C. C., Ergodic equivalence relations, cohomology, and von Neumann algebras. I, Trans. Amer. Math. Soc., 234 (1977), 289-324.

[7] Hahn, P., The regular representations of measure groupoids, Trans. Amer. Math. Soc., 242 (1978), 35-72. 
[8] - Reconstruction of a factor from measures on Takesaki's unitary equivalence relation, J. Functional Analysis, 31 (1979), 263-271.

[9] Mackey, G. W., Borel structure in groups and their duals, Trans. Amer. Math. Soc., 85 (1957), 134-165.

[10] Ramsay, A., Virtual groups and group actions, Advances in Math., 6 (1971), 253322.

[11] Strătilă, Ș. and Zsidó, L., Lectures on von Neumann algebras, Editura Academiei and Abacus Press, Romania and England, 1979.

[12] Takesaki, M., On the unitary equivalence among the components of decompositions of representations of involutive Banach algebras and the associated diagonal algebras, Tôhoku Math. J., 15 (1963), 365-393.

[13] Zimmer, R. J., Strong rigidity for ergodic actions of semisimple Lie groups, Ann. of Math., 112 (1980), 511-529. 
